\title{
Intravenous fluid prescribing for medical inpatients: are we getting it right?
}

\author{
Authors: Agrima Ghosh and Carlos Maltez
}

\section{Introduction}

Intravenous fluids (IVF) are commonly prescribed for hospital inpatients by junior members of the medical team. There is significant morbidity and mortality associated with inappropriate IVF prescribing secondary to iatrogenic fluid, electrolytes and glucose derangement. The National Institute for Health and Care Excellence (NICE) clinical guideline CG174 defines the amount of water and electrolytes patients should receive during IVF administration for routine maintenance to prevent biochemical derangement. We aimed to assess our adherence to the guideline.

\section{Materials and methods}

Over 5 days, patients on a single ward who required intravenous fluids for routine maintenance only were included in this study. Patients who were pregnant, critically unwell, had diabetes, severe renal or liver impairment, or abnormal gastrointestinal loss were excluded from this study. The daily amount of IVF and constituents (sodium, potassium, chloride and glucose) provided to the patient was calculated. This was compared with that advised in the NICE guideline.

\section{Results and discussion}

Twenty-three patients were identified during the 5 days that satisfied the inclusion criteria. The data for the IVF each patient received was collected over 24 hours. There were 15 males and eight females with an average (range) age of 67 (23-91) years and average (range) weight of $72.3 \mathrm{~kg}(44.8-100.2 \mathrm{~kg})$. Sodium chloride $(0.9 \%)$, Hartmann's fluids, dextrose-saline, sodium chloride plus Hartmann's, and dextrose-saline plus Hartmann's were used in $65.2 \%, 13.0 \%, 4.3 \%, 13 \%$ and $4.3 \%$, respectively. The average (range) delivery of water was 33.48 (14.7-46.2) mL/kg/day; sodium: 4.63 (2.21-6.67) mmol/kg/day; potassium: 0.15 (0.05-0.23) $\mathrm{mmol} / \mathrm{kg} /$ day; chloride: 4.54 (2.24-5.94) $\mathrm{mmol} / \mathrm{kg} /$ day; glucose: 70 (59-80) g/day. Fifteen patients were given more water than recommended, all patients were given more sodium and chloride than recommended, and all were given less potassium than recommended. Only two patients were given glucose and these were as recommended.

\section{Conclusion}

This study demonstrates that we are not providing our patients with the right amount of water and electrolytes over 24 hours when prescribing IVF for routine maintenance. Extensive education is required to improve the thought process during IVF prescribing among junior doctors. This is especially important during the early years of training, as it is the foundation for future clinical practice. The points that can be highlighted during teaching sessions include advising prescribing fluids required for the full 24 hours to avoid the on-call team to be called, daily urea and electrolytes to be checked while the patient is on IVF, and potentially using input/output charts to monitor fluid balance for such patients. The importance of an IVF specialist team cannot be overemphasised.

\section{Reference}

1 National Institute for Health and Care Excellence. Intravenous fluid therapy in adults in hospital. London: NICE, 2013. Available at: www. nice.org.uk/guidance/cg174/chapter/1-Recommendations\#routinemaintenance-2 [Accessed 23 February 2019]. 